\title{
Engine gearbox fault diagnosis using empirical mode decomposition method and Naïve Bayes algorithm
}

\author{
KIRAN VERNEKAR, HEMANTHA KUMAR* and K V GANGADHARAN \\ Department of Mechanical Engineering, National Institute of Technology Karnataka, \\ Surathkal, Mangalore 575025, India \\ e-mail: hemanta76@gmail.com
}

MS received 15 September 2014; revised 24 September 2016; accepted 19 January 2017

\begin{abstract}
This paper presents engine gearbox fault diagnosis based on empirical mode decomposition (EMD) and Naïve Bayes algorithm. In this study, vibration signals from a gear box are acquired with healthy and different simulated faulty conditions of gear and bearing. The vibration signals are decomposed into a finite number of intrinsic mode functions using the EMD method. Decision tree technique (J48 algorithm) is used for important feature selection out of extracted features. Naïve Bayes algorithm is applied as a fault classifier to know the status of an engine. The experimental result (classification accuracy 98.88\%) demonstrates that the proposed approach is an effective method for engine fault diagnosis.
\end{abstract}

Keywords. Engine fault diagnosis; empirical mode decomposition; decision tree technique; Naïve Bayes.

\section{Introduction}

Machine elements such as a gear, bearing and shaft play an important role in the gearbox of vehicle transmission system. Most of the defects in transmission system are generated due to fatigue, excessive loads and improper lubrication. Defects occasionally arise during the assembly process in production line. These faults in transmission system may cause excessive vibration, noise and even failure of transmission system. In an engine manufacturing industry, it is necessary to identify and eliminate the defective engines going into the market [1]. The present study focuses on fault diagnosis of an internal combustion (IC) engine gearbox using machine learning techniques. The vibration signals are acquired from the engine gear box. These original vibration signals are decomposed using empirical mode decomposition (EMD) and the first few important intrinsic mode functions (IMFs) are obtained. Amplitude energy features of the stationary IMFs are evaluated and treated as an input feature vector to the J48 algorithm for important feature selection. To distinguish the healthy from the simulated faulty condition of gears and bearings, Naïve Bayes algorithm is used as a classifier.

A lot of research has been carried out by investigations on fault diagnosis of machinery system using signal processing and artificial intelligence techniques. Chen et al [2] carried out investigation on gearbox fault diagnosis using the EMD method to extract features from

*For correspondence vibration signal. They used the immune genetic algorithm for important features selection out of extracted features and a wavelet support vector machine for fault classification. Yu and Junsheng [3] investigated fault diagnosis of a roller bearing using the EMD method and used artificial neural network (ANN) as a classifier. Kumar et al [4] employed the Naïve Bayes classifier to perform fault diagnosis of bearing through vibration signals. Many researchers implemented Naïve Bayes as a fault classifier in the area of damage detection in engineering materials [5] and centrifugal pumps [6] and mechanical rotary machine faults diagnosis of components such as gears [7] and bearings [8]. Sharma et al [9] conducted experiments to identify the effectiveness of J48 algorithm for misfire detection of IC engine. A few studies were reported based on decision tree algorithm for mechanical rotary machine faults diagnosis [10-13]. Wang et al [14] carried out an experiment on engine fault diagnosis through sound signals using the HilbertHuang transform for feature extraction and a support vector machine used for classification. The literature on fault diagnosis of an IC engine gearbox based on machine learning approach is very limited. Investigation on engine gear and bearing conditions is very essential in fault diagnosis of gearbox system. This paper deals with the analysis of different conditions of gear and bearing of two-stroke IC engine gearbox using vibration signals. In this work the EMD method, decision tree and Naïve Bayes algorithm have been adopted for feature extraction, feature selection and fault classification of gear and bearing using vibration signals. 


\section{Experimental studies}

An engine test rig has been developed to study fault diagnosis of mechanical components used in IC engine. The details about the experimental set-up and procedure are discussed in the following subsections.

\subsection{Experimental set-up description}

Figures 1 and 2 illustrate the line diagram and experimental test set-up used for fault detection of IC engine gearbox. The experiments were conducted on a Kawasaki Bajaj (KB-100) two-stroke spark ignition engine without considering the combustion. Generally, the engine conditions are monitored at idle speed, which is near $1000 \mathrm{rpm}$. Therefore, the experiment has been carried out at constant rotational engine crank speed of $1100 \mathrm{rpm}$ at 4 th gear position. The engine and motor were mounted on a test bed as shown in figure 2. Vibration isolation rubber pads were provided under the test bed to reduce the vibration transmission from ground to the test bed. The engine was driven using a $3 \mathrm{hp} \mathrm{DC}$ motor. The drive to the induction motor was delivered by a DC power supply.

A piezoelectric accelerometer with an operating frequency range between 1 and $5000 \mathrm{~Hz}$ was used to pick up vibration signals (magnitude of acceleration with respect to time). The sampling frequency of $25.6 \mathrm{kHz}$ was used to collect data for $1 \mathrm{~s}$. The data were acquired using a NI-9234 data acquisition (DAQ) card and analysed using LabVIEW software from

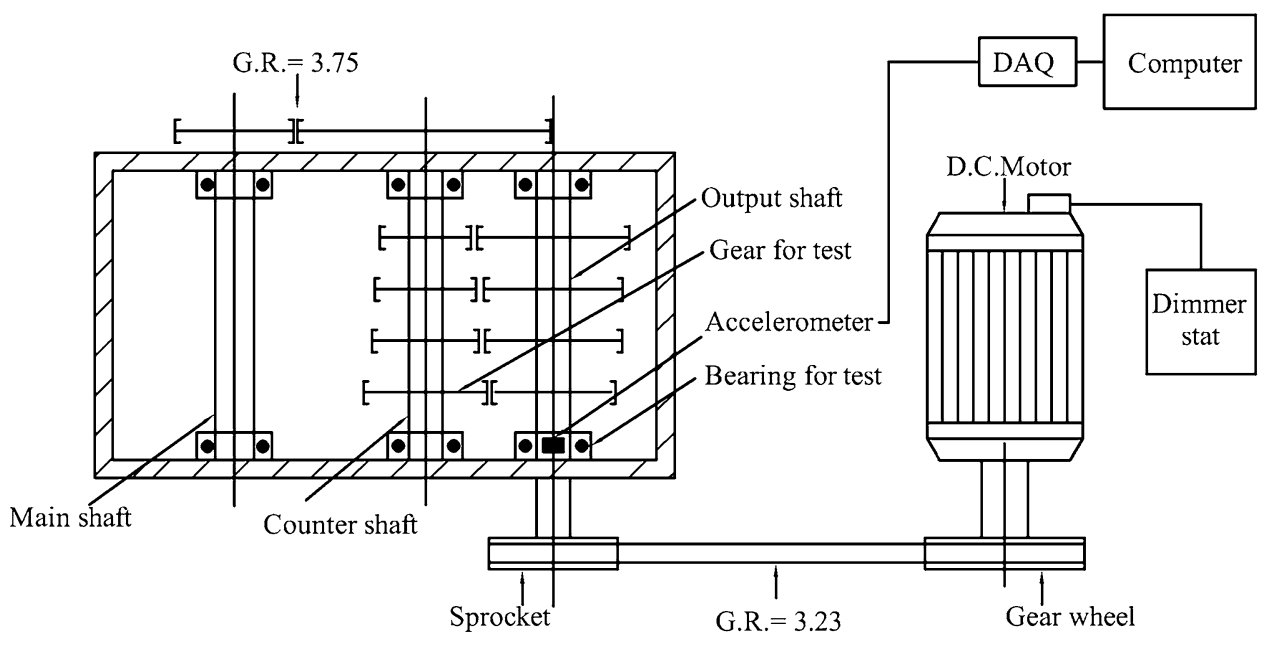

Figure 1. Line diagram of experimental set-up.

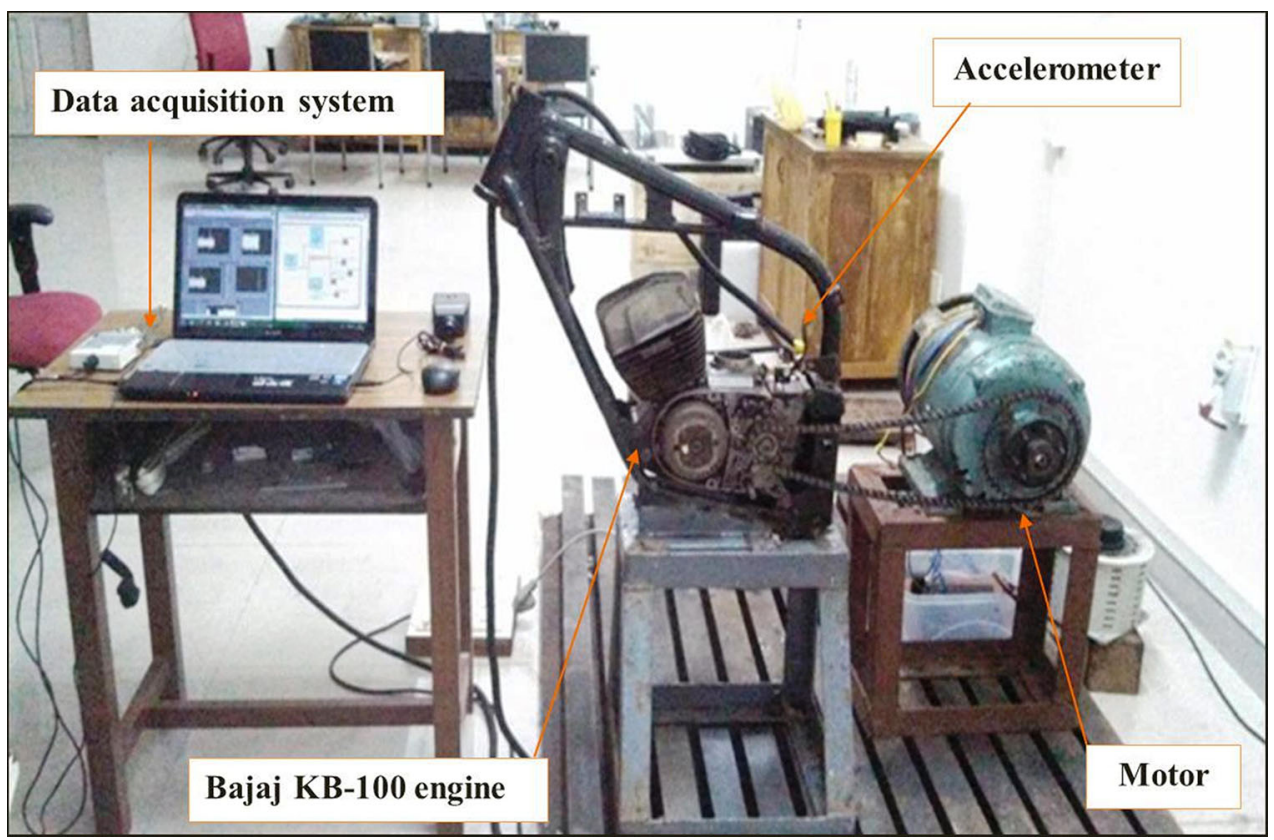

Figure 2. IC engine fault diagnosis test set-up. 


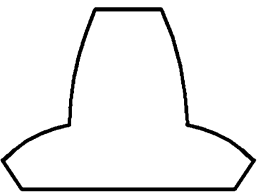

(a) Healthy gear

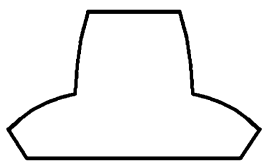

(b) $25 \%$ faulty gear

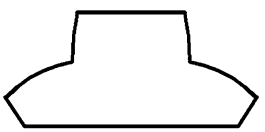

(c) $50 \%$ faulty gear

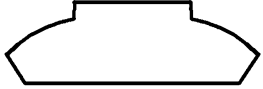

(d) $75 \%$ faulty gear

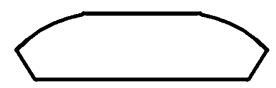

(e) $100 \%$ faulty gear

Figure 3. Gear tooth profiles.

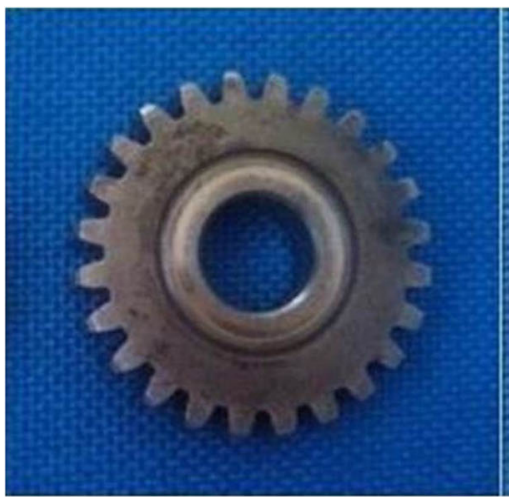

(a) Healthy gear

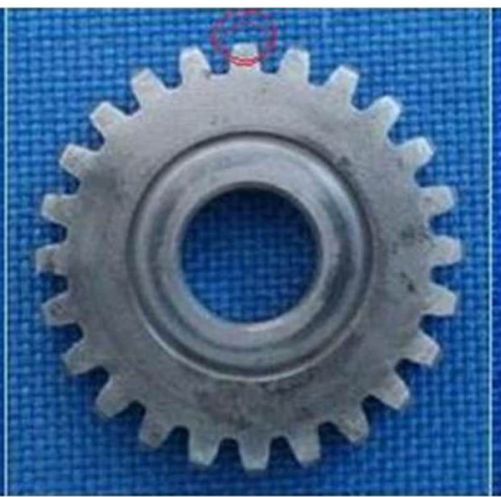

(b) $25 \%$ faulty gear

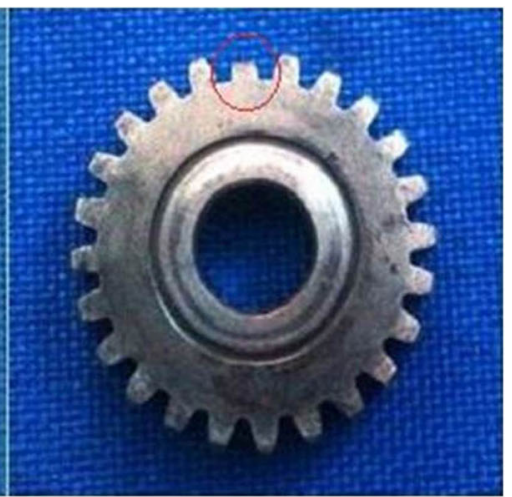

(c) $50 \%$ faulty gear

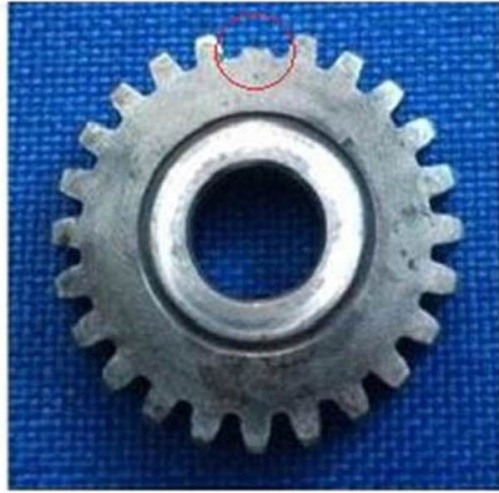

(d) $75 \%$ faulty gear

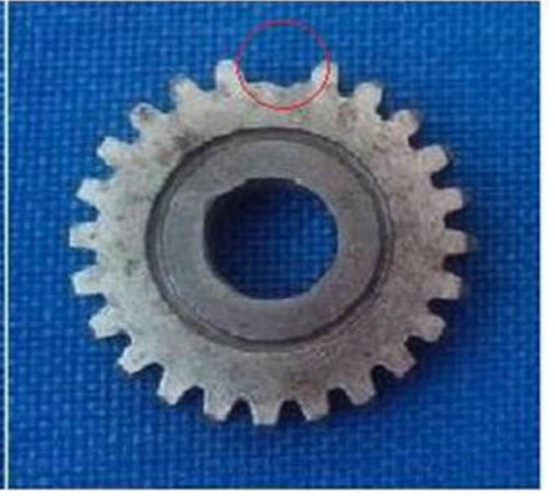

(e) $100 \%$ faulty gear

Figure 4. Different conditions of gear.

National Instruments (NI). Output from the accelerometer was fed to DAQ to convert measured physical parameters to electrical signals. A DAQ system consists of sensors, DAQ measurement hardware and a computer with programmable software. A LabVIEW software Virtual Instrument (VI) program was developed to acquire and analyse vibration data for these experiments. In order to avoid inconsistency in DAQ, three trials were performed at the set speed and vibration signal was stored in the data file.

\subsection{Experimental procedure}

In this study, artificial defects were introduced in IC engine components, namely gear and bearing for engine fault diagnosis. In case of gear, artificial defects were introduced by machining the gear tooth progressively by $25,50,75$ and $100 \%$ (one tooth removal) of the tooth face width as shown in figure 3. These simulated faults resemble the practical damaged gear condition generated due to fatigue, excessive loads, eccentric teeth, etc. The different conditions of gear used in this experiment are shown in figure 4 . In case of bearing, a deep groove ball bearing (6004) on gear box casing cover side has been considered for fault diagnosis study. Artificial faults were generated at different locations of the bearing and they are bearing outer race, inner race, inner and outer race together fault and rolling element (ball) fault. In the case of inner race fault, while transmitting impulse to the outer surface of the casing, inner-race damage has more transfer segments. These impulse components are weak in the vibration signal. Therefore, diagnosis for inner-race fault is very difficult [15]. Therefore, to 


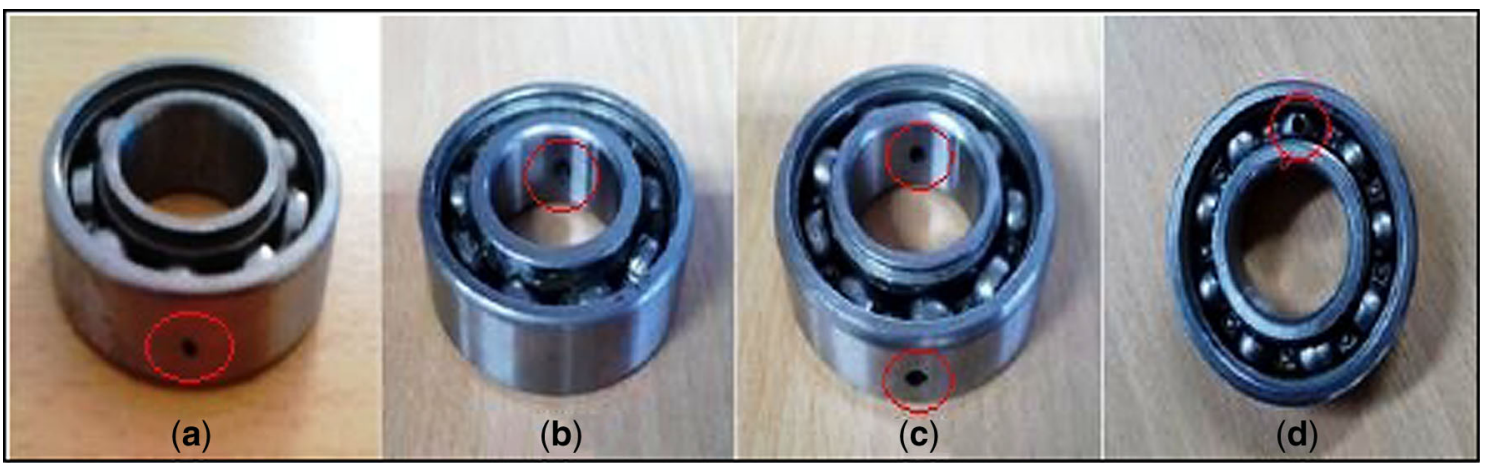

Figure 5. Bearing defects: (a) outer race defect, (b) inner race defect, (c) inner and outer race defect and (d) ball defect.

Table 1. Experimental condition of gears and bearings.

\begin{tabular}{lcc}
\hline Cases & Severity of fault & Samples collected \\
\hline $\mathrm{a}$ & Healthy condition & 30 \\
$\mathrm{~b}$ & $25 \%$ tooth removal & 30 \\
$\mathrm{c}$ & $50 \%$ tooth removal & 30 \\
$\mathrm{~d}$ & $75 \%$ tooth removal & 30 \\
$\mathrm{e}$ & $100 \%$ tooth removal & 30 \\
$\mathrm{f}$ & $1.5 \mathrm{~mm}$ outer race fault & 30 \\
$\mathrm{~g}$ & $2.5 \mathrm{~mm}$ inner race fault & 30 \\
$\mathrm{~h}$ & $2.5 \mathrm{~mm}$ inner and outer race fault & 30 \\
$\mathrm{i}$ & $2 \mathrm{~mm}$ ball fault & 30 \\
\hline
\end{tabular}

exaggerate the case of inner race defect, a hole of $2.5 \mathrm{~mm}$ was drilled on it. The defective bearings are shown in figure 5. Table 1 lists different conditions or classes of gears and bearing for which engine gearbox was tested. In all, 30 vibration signals pertaining to each condition of gearboxes were acquired as explained in section 2.1. Figure 6 shows the position of the gear and bearing in the gear box.

Time domain plots representing the dynamic responses depend on the interaction of many components of the system. One of the acquired vibration signal samples in time domain for each condition of the gear and bearing is shown in figure 7 . From time domain signals it was observed that the acceleration level increases with increase in severity of fault.

\section{Machine learning approach in fault diagnosis}

The methodology involved in the fault diagnosis of engine with machine learning approach is illustrated in figure 8. Vibration signal from the engine gear box was acquired using an accelerometer. In all, 30 vibration signal samples were collected for each condition as mentioned in table 1. The first eight IMFs containing

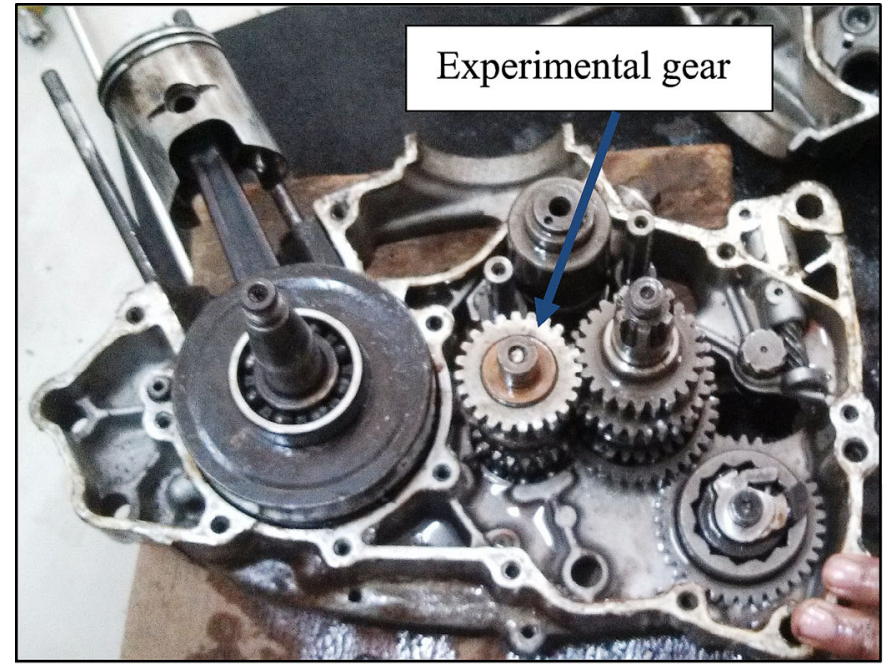

(a)

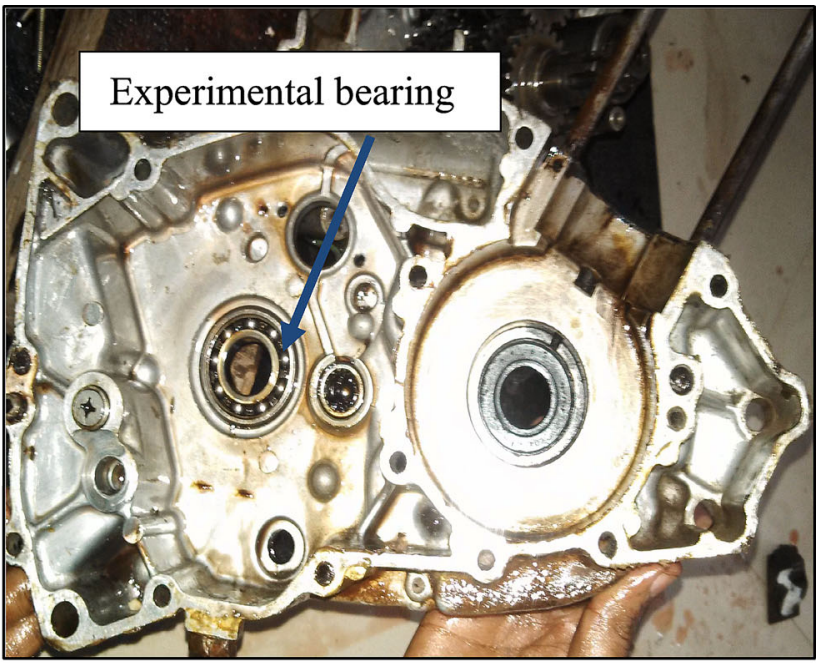

(b)

Figure 6. Sectioned view of engine. 


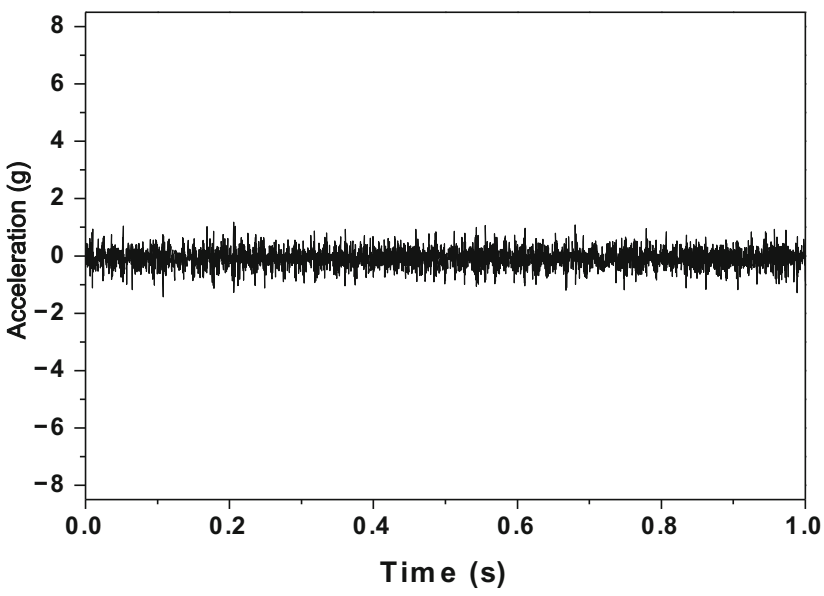

(a) Healthy condition

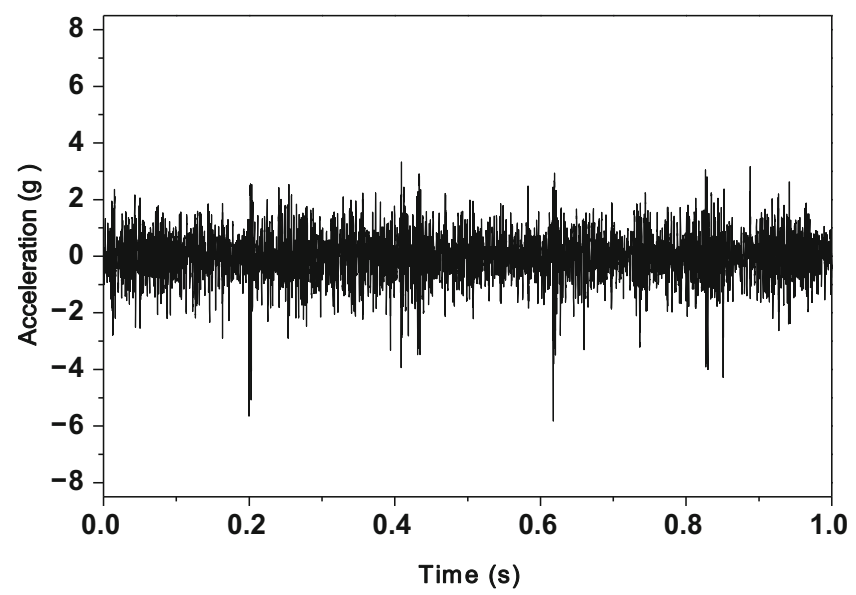

(c) $50 \%$ faulty gear

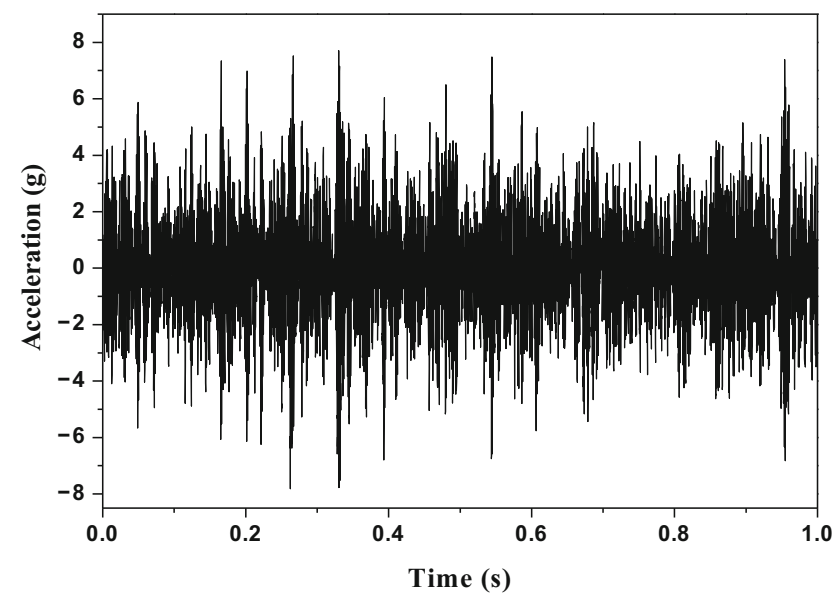

(e) $100 \%$ faulty gear

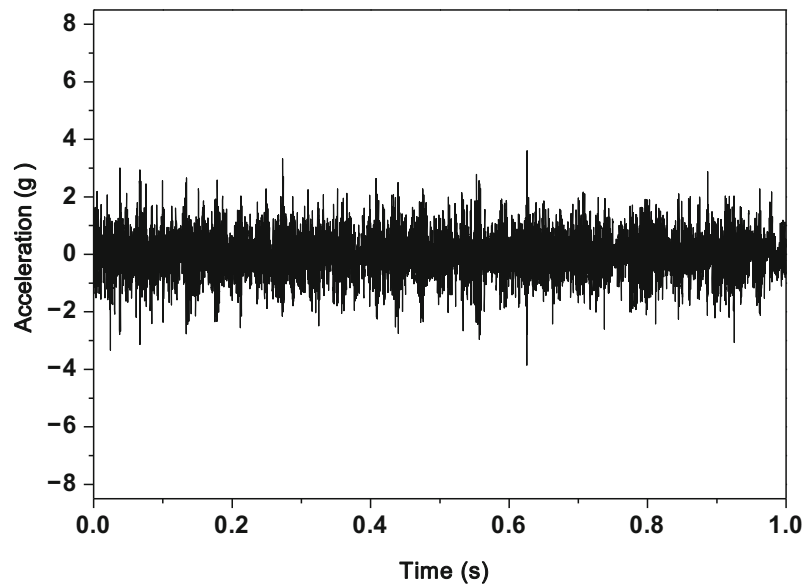

(b) $25 \%$ faulty gear

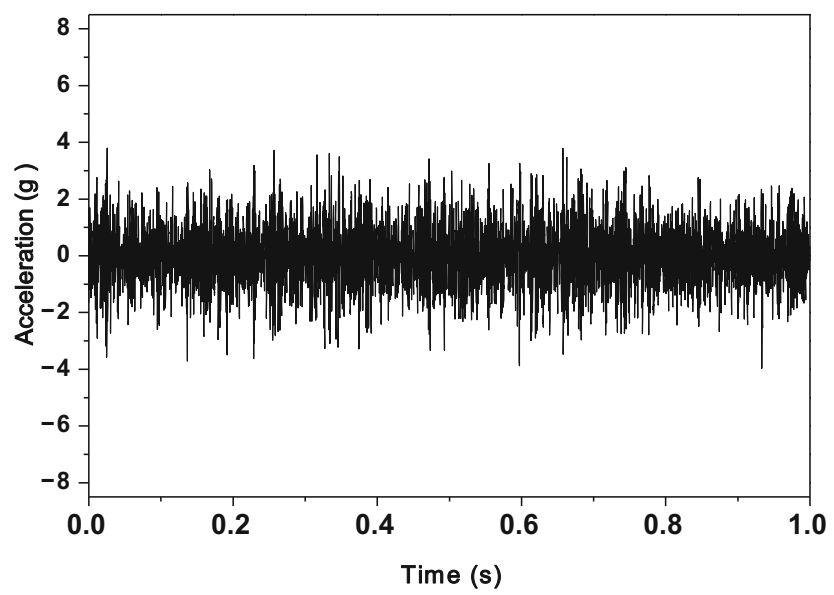

(d) $75 \%$ faulty gear

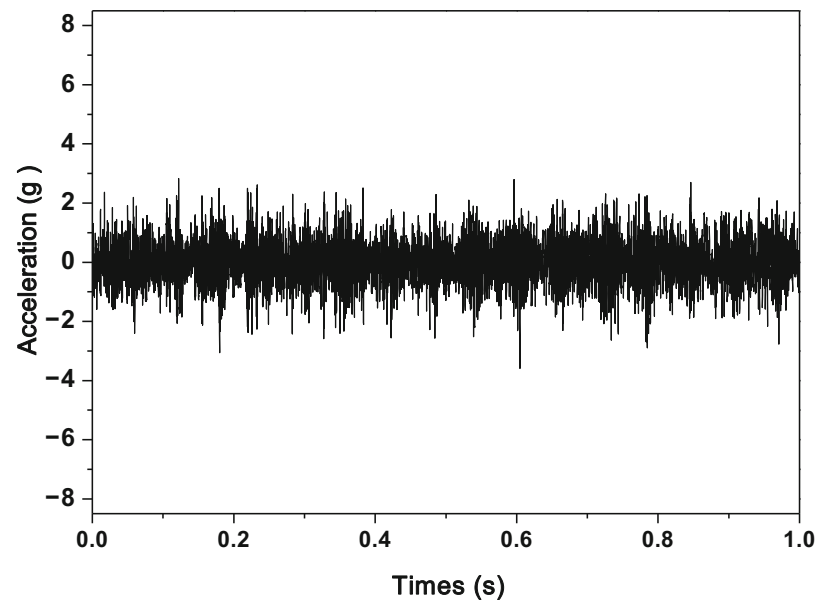

(f) Outer race fault bearing

Figure 7. Vibration response in time domain.

validation information of original signal were decomposed using the EMD method. Energy feature vector was evaluated and treated as input vector to J48 algorithm for important feature selection and fault classification using the Naïve Bayes algorithm.

\section{EMD feature extraction}

The assumption behind the development of EMD algorithm is that any signal consists of different intrinsic modes of oscillations. EMD simply decomposes a non-stationary 


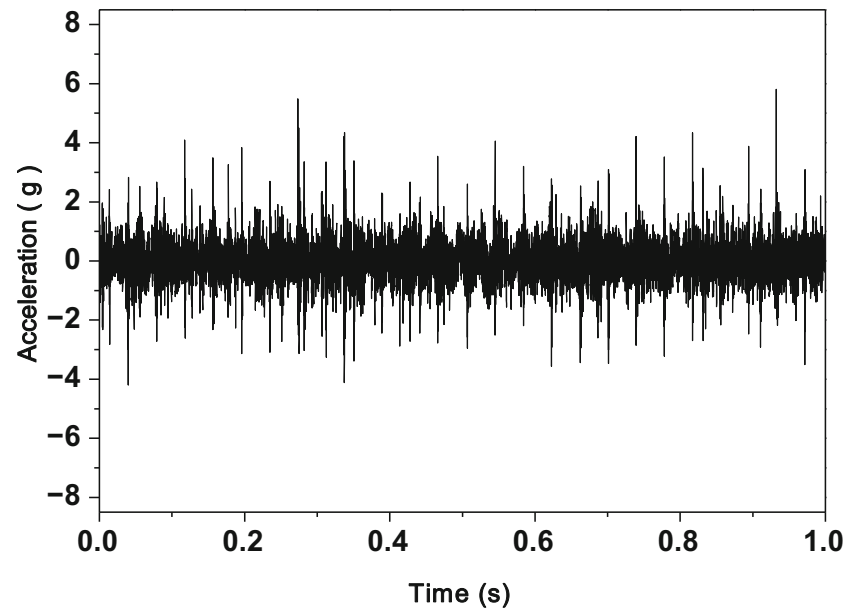

(g) Inner race defect bearing

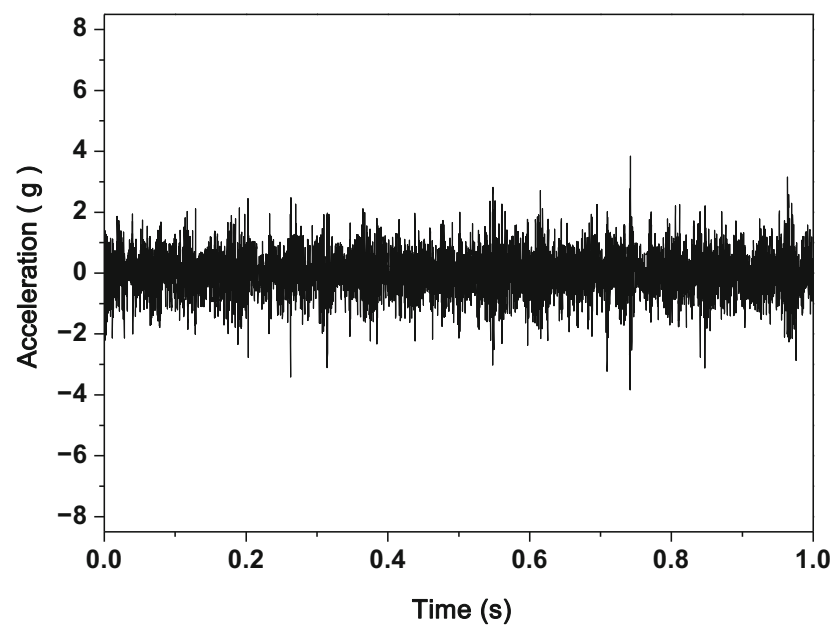

(h) Inner and outer race defect bearing

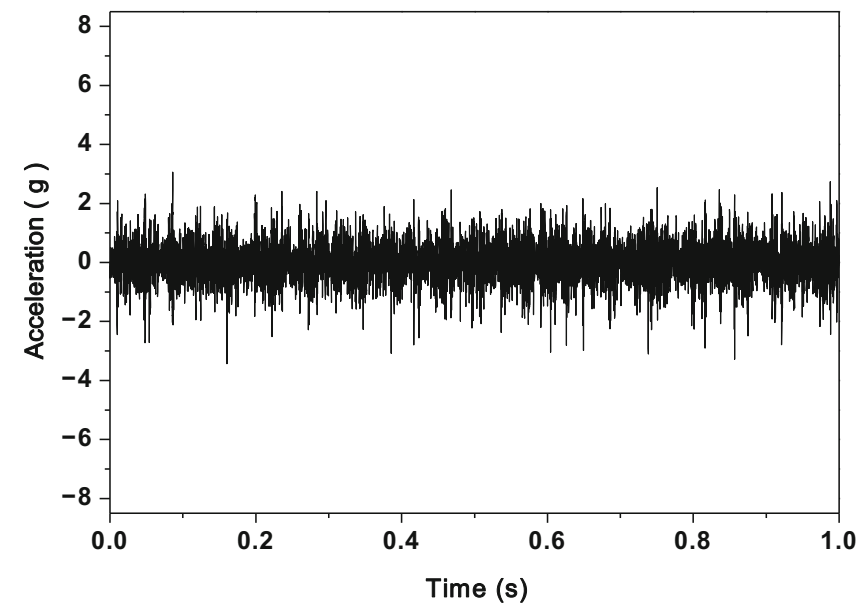

(i) Ball defect bearing

Figure 7. continued

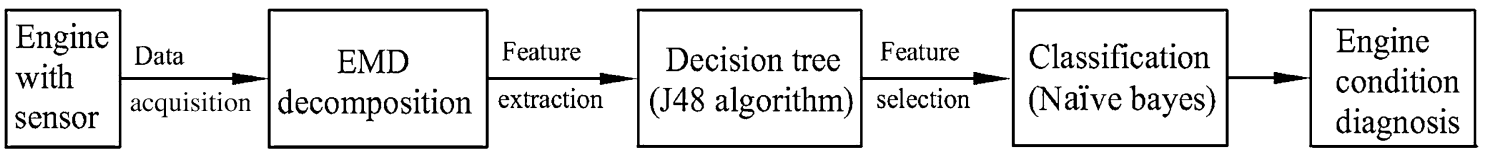

Figure 8. Flow chart of engine fault diagnosis with machine learning approach.

signal into some IMFs. An IMF is a function that satisfies the two following conditions:

1. The number of IMF extrema (the sum of the maxima and minima) and the number of zero-crossings must either be equal or differ at most by one;

2. At any point of an IMF, the mean value of the envelope defined by the local maxima and the envelope defined by the local minima shall be zero.

An IMF represents a simple oscillatory mode compared to the simple harmonic function. With the dentition, any signal $x(t)$ can be decomposed as follows:

$$
x(t)=\sum_{i=1}^{n} c_{i}(t)+r_{n} .
$$

EMD decomposes the given signal into $n$ empirical modes and a residue $r_{n}$, which represents the central tendency of signal $x(t)$. The IMFs $c_{1}(t), c_{2}(t), \ldots, c_{n}(t)$ include different frequency bands ranging from high to low. The frequency components contained in each frequency band are different and they change with the variation of signal $x(t)$.

The EMD algorithm decomposes the signal $x(t)$ into a number of IMFs and each resulting IMF component consists of the local characteristic of the signal. When 
the gearbox with different mechanical faults operates, the amplitude energy of each IMF is obviously different. Therefore, amplitude energy feature of each IMF component can be used to identify the different faults of mechanical components of an IC engine gear box. In order to classify the faults, the amplitude energy feature can be treated as the input vector for decision tree. According to the principle of EMD, the original signal can be decomposed into $n$ empirical modes and a residue $r_{n}$; the total energy $E_{i}$ of the first $n$ IMFs is calculated as

$$
E_{n i}=\sum_{i=1}^{m}\left|c_{i}(t)\right|^{2}
$$

where $E_{n i}$ represents amplitude energy of the $i$ th IMF and ' $m$ ' indicates the discrete data length of the $i$ th IMF. A feature vector with the amplitude energy as element is constructed as

$$
T=\left\{E_{n 1}, E_{n 2}, \ldots, E_{n m}\right\}
$$

where ' $n$ ' represents the number of IMFs.

Considering that some IMF energy maybe sometimes very large, it is necessary to avoid attributes in larger numeric ranges dominating those in smaller numeric ranges and reduce numerical difficulties during the calculation. $T$ is regulated by normalizing the feature for the convenience of the following analysis and processing. Let $E=\left(\sum_{i=1}^{n} \mid-\right.$ $\left.\left.E_{n i}\right|^{2}\right)^{1 / 2}$ and

$$
T^{\prime}=\left\{\frac{E_{n 1}}{E}, \frac{E_{n 2}}{E}, \ldots, \frac{E_{n m}}{E}\right\}=\left\{E_{1}, E_{2}, \ldots, E_{n}\right\}
$$

where $T^{\prime}$ represents the normalized vector. This normalized vector $T^{\prime}$ shall be treated as an input vector of the proposed fault diagnosis model so that gearbox mechanical fault can be distinguished [2].

\section{Feature selection using decision tree}

The features are measures of data points or signals. The process of selecting useful features from the extracted features is termed as 'feature selection'. The decision tree consists of a tree-structured algorithm that depicts the classification information of the data. The purpose of decision tree is to expose the information contained in the data. It is a predictive machine-learning model. As the name indicates, decision tree follows the pattern of a tree that is constructed using a single root, number of branches, number of nodes and leaves. The branch that starts from the root to leaf is a chain of nodes and each node consists of one attribute. The occurrence of an attribute is the number of times the attribute occurs in the tree, which provides the information about the importance of the associated attribute [16].
The method of forming the decision tree and exploiting the same for feature selection is given as follows:

- The group of features obtained by EMD is treated as input to the decision tree algorithm.

- The decision tree consists of leaf nodes, which indicate class labels, and other nodes accompanied with the classes to be classified.

- Each branch of the tree illustrates each possible value of the feature node from which it is generated.

- The decision tree can be used to classify feature vectors by starting at the root to leaf node of the tree, which provides a classification of the instance and is identified.

- At each decision node, one can select the most convenient feature for classification using appropriate estimation criteria. This selection brings about the concepts of entropy reduction and information gain, which are discussed in the following subsections [17].

\subsection{Information gain and entropy reduction}

Information gain is used for measuring association between inputs and outputs. It is a state to state change in information entropy. Entropy is a measure of disorder of data. It is generated from information theory. As the entropy value increases, it will get more information content. For feature ' $A$ ', information gain $(S, A)$ relative to a collection of examples $S$ is defined as follows:

$$
\operatorname{gain}(S, A)=\operatorname{entropy}(S)-\sum_{v \in \text { value }(A)} \frac{\left|S_{v}\right|}{|S|} \operatorname{entropy}\left(s_{v}\right)
$$

where value (A) is the set of all possible values for attribute $A$ and ' $S_{v}$ ' is the subset of $S$ for which feature $A$ has value $v$.

Entropy is given by the relation

$$
\operatorname{entropy}(S)=\sum_{i=1}^{c}-P_{i} \log _{2} P_{i}
$$

where ' $c$ ' represents the number of classes and ' $P_{i}$ ' is probability of class ' $i$ ', computed as the proportion of class ' $i$ ' in the set.

\section{Naïve Bayes classifier}

The Naïve Bayes algorithm was developed based on the assumptions that all variables in the network are independent of the classification variable, i.e., attributes $X_{1}, \ldots, X_{n}$ are all conditionally independent of each other, given $Y$. The value of this assumption is that it dramatically simplifies the representation of $P(X \mid Y)$ and the problem of estimating it from the training data. Let us consider a case where $X=\left(X_{1}, X_{2}\right)$ [4]. In this case 


$$
\begin{aligned}
P(X \mid Y) & =P\left(X_{1}, X_{2} \mid Y\right)=P\left(X_{1} \mid X_{2}, Y\right) P\left(X_{2} \mid Y\right) \\
& =P\left(X_{1} \mid Y\right) P\left(X_{2} \mid Y\right) .
\end{aligned}
$$

More generally, when $X$ contains $n$ attributes that are conditionally independent of one another given $Y$, we have

$$
P\left(X_{1}, \ldots, X_{n} \mid Y\right)=\prod_{i=1}^{n} P\left(X_{i} \mid Y\right) .
$$

When $X_{i}$ and $Y$ are Boolean variables, we need only $2 n$ parameters to define $P\left(X_{i}=X_{i k} \mid Y=y_{j}\right)$ for the necessary $i, j, k$. This is a substantial reduction when compared with $2\left(2^{n}-1\right)$ parameters needed to characterize $P(X \mid Y)$ if we make no conditional independence assumption.

Let us now derive the Naïve Bayes algorithm, assuming in general that $Y$ is any discrete-valued variable and the attributes $X_{1}, \ldots, X_{n}$ are any discrete or real-valued attributes. Our goal is to train a classifier that will output the probability distribution over the possible values of $Y$, for each new instance $X$ that we ask it to classify. The expression for the probability that $Y$ will take on its $k$ th possible value, according to the Bayes rule, is

$P\left(Y=y_{k} \mid X_{1}, \ldots, X_{n}\right)=\frac{P\left(Y=y_{k}\right) P\left(X_{1}, \ldots, X_{n} \mid Y=y_{k}\right)}{\sum_{j} P\left(Y=y_{j}\right) P\left(X_{1}, \ldots, X_{n} \mid Y=y_{j}\right)}$.

The sum is taken over all possible values of $y_{j}$ of $Y$. Assuming that $X_{i}$ is conditionally independent given $Y$, we can use Eq. (9) to rewrite this as

$$
P\left(Y=y_{k} \mid \mathrm{X}_{1}, \ldots, X_{n}\right)=\frac{P\left(Y=y_{k}\right) \prod_{i} P\left(\mathrm{X}_{i} \mid \mathrm{Y}=\mathrm{y}_{\mathrm{k}}\right)}{\sum_{j} P\left(Y=y_{j}\right) \prod_{i} P\left(\mathrm{X}_{i} \mid Y=y_{j}\right)} .
$$

Equation (10) is the fundamental equation for the Naïve Bayes classifier. Given a new instance $X_{\text {new }}=\left(1, \ldots, X_{n}\right)$, this equation shows how to calculate the probability that $Y$ will take on any given value, given the observed attribute values of $X_{\text {new }}$ and given the distributions $P(Y)$ and $P\left(X_{i} \mid Y\right)$ estimated from the training data [6]. If the most probable value of $Y$ is to be found, then the Naïve Bayes classification rule is given by

$$
y \leftarrow \arg \max y_{k} \frac{P\left(Y=y_{k}\right) \prod_{i} P\left(X_{i} \mid \mathrm{Y}=\mathrm{y}_{\mathrm{k}}\right)}{\sum_{j} P\left(Y=y_{j}\right) \prod_{i} P\left(X_{i} \mid Y=y_{j}\right)}
$$

which simplifies to the following (because the denominator does not depend on $y_{k}$ ):

$$
y \leftarrow \arg \max y_{k} P\left(Y=y_{k}\right) \prod_{i} P\left(X_{i} \mid Y=y_{k}\right) .
$$

\subsection{Bayes net}

A Bayesian network is a probabilistic graphical model. It consists of a set of variables, $V=\left\{A_{1}, A_{2}, \ldots, A_{N}\right\}$, and a set of directed edge, $E$, between variables, which form a directed acyclic graph (DAG) $G=(V, E)$, where a joint distribution of variables is represented by the product of conditional distributions of each variable given its parents. Each node $A_{i} \in V$ represents a random variable and a directed edge from $A_{i}$ to $A_{j},\left(A_{i}, A_{j}\right) \in V$, represents the conditional dependency between $A_{i}$ and $A_{j}$. In a Bayesian network, each variable is independent of its non-descendants, given a value of its parents in $G$. This independence encoded in $G$ reduces the number of parameters required to characterize a joint distribution, so that posterior distribution can be efficiently inferred. In a Bayesian network, over $V=\left\{A_{1}, A_{2}, \ldots, A_{N}\right\}$, the joint distribution $P(V)$ is the product of all conditional distributions specified in the Bayesian network such as

$$
P\left(A_{1}, A_{2}, \ldots, A_{N}\right)=\prod_{i=1}^{N} P\left(A_{i} \mid \mathrm{Pa}_{i}\right)
$$

where $P\left(A_{i} \mid \mathrm{Pa}_{i}\right)$ is the conditional distribution of $A_{i}$, given $\mathrm{Pa}_{i}$, which denotes the parent set of $A_{i}$. A conditional distribution for each variable has a parametric form that can be learned by the maximum likelihood estimation [18].

\section{Experimental results and analysis}

The vibration signals were acquired using an accelerometer mounted on the gearbox, while keeping motor speed at $1100 \mathrm{rpm}$ and the sampling frequency at $25.6 \mathrm{kHz}$. In all, 30 samples for each state were acquired for nine different conditions of engine gearbox. Therefore, 270 samples in total, including the normal condition, were collected for further analysis. After preprocessing, the acquired vibration signal is decomposed into some IMFs by the EMD method. The first eight IMFs, which contain the dominant fault information, were used to construct the amplitude energy feature vector. Feature energy vector $T^{\prime}$ was obtained from Eq. (4) and treated as an input vector to the decision tree (J48 algorithm). Naïve Bayes model has been used as a classifier to categorize the fault conditions of engine gearbox.

\subsection{Feature extraction and selection}

Table 2 shows the evaluated energy feature vector using the EMD method. Out of 30 samples, due to limited space, only two samples pertaining to each class of gear and bearing are shown in this table. From this table, it can be observed that it is possible to distinguish the samples since all features for each class are different to some extent. However, in an online condition-monitoring cell, it becomes tedious work for an operator to distinguish the difference, for fault diagnosis and the diagnosis result may be unreliable.

Therefore, important features out of extracted features are selected using decision tree (J48 algorithm). Figure 9 illustrates the decision tree generated by J48 algorithm, which 
Table 2. Feature vector based on EMD method.

\begin{tabular}{|c|c|c|c|c|c|c|c|c|c|}
\hline \multirow[b]{2}{*}{ Class } & \multirow[b]{2}{*}{ Sample no. } & \multicolumn{8}{|c|}{ Energy feature vector } \\
\hline & & $E 1$ & $E 2$ & $E 3$ & $E 4$ & $E 5$ & E6 & E7 & $E 8$ \\
\hline \multirow[t]{2}{*}{ Healthy } & 1 & 0.4427 & 0.1186 & 0.0449 & 0.0395 & 0.0332 & 0.0199 & 0.0109 & 0.4427 \\
\hline & 2 & 0.3857 & 0.1245 & 0.0387 & 0.0218 & 0.0146 & 0.0177 & 0.0222 & 0.3857 \\
\hline \multirow[t]{2}{*}{$25 \%$ tooth removal } & 1 & 0.9776 & 0.1072 & 0.0615 & 0.0328 & 0.0417 & 0.0211 & 0.0795 & 0.1395 \\
\hline & 2 & 0.9776 & 0.1072 & 0.0615 & 0.0328 & 0.0417 & 0.0211 & 0.0795 & 0.1395 \\
\hline \multirow[t]{2}{*}{$50 \%$ tooth removal } & 1 & 0.9713 & 0.1779 & 0.0784 & 0.038 & 0.0215 & 0.0267 & 0.0865 & 0.0928 \\
\hline & 2 & 0.9715 & 0.184 & 0.0852 & 0.0295 & 0.0236 & 0.0206 & 0.0104 & 0.0054 \\
\hline \multirow[t]{2}{*}{$75 \%$ tooth removal } & 1 & 0.9961 & 0.0407 & 0.0216 & 0.0132 & 0.0169 & 0.02 & 0.0448 & 0.0529 \\
\hline & 2 & 0.9939 & 0.0458 & 0.0265 & 0.0103 & 0.0134 & 0.0386 & 0.0782 & 0.0382 \\
\hline \multirow[t]{2}{*}{$100 \%$ tooth removal } & 1 & 0.9406 & 0.2983 & 0.1361 & 0.0553 & 0.0207 & 0.0139 & 0.0138 & 0.0625 \\
\hline & 2 & 0.9406 & 0.2983 & 0.1361 & 0.0553 & 0.0207 & 0.0139 & 0.0138 & 0.0625 \\
\hline \multirow[t]{2}{*}{ Outer race defect } & 1 & 0.9182 & 0.227 & 0.0718 & 0.0396 & 0.018 & 0.0298 & 0.0565 & 0.307 \\
\hline & 2 & 0.9179 & 0.2033 & 0.0735 & 0.0481 & 0.0558 & 0.2309 & 0.2097 & 0.0896 \\
\hline \multirow[t]{2}{*}{ Inner race defect } & 1 & 0.9449 & 0.2432 & 0.0849 & 0.0643 & 0.0687 & 0.0667 & 0.0517 & 0.1573 \\
\hline & 2 & 0.9547 & 0.2523 & 0.0793 & 0.0514 & 0.0197 & 0.0124 & 0.0212 & 0.1228 \\
\hline \multirow[t]{2}{*}{ Inner and outer race defect } & 1 & 0.9298 & 0.1945 & 0.0613 & 0.036 & 0.0159 & 0.0154 & 0.0401 & 0.3007 \\
\hline & 2 & 0.9024 & 0.2125 & 0.0671 & 0.0416 & 0.0187 & 0.0134 & 0.0201 & 0.3651 \\
\hline \multirow[t]{2}{*}{ Ball defect } & 1 & 0.9684 & 0.2341 & 0.0758 & 0.0368 & 0.0114 & 0.0046 & 0.0032 & 0.0052 \\
\hline & 2 & 0.9593 & 0.2587 & 0.0843 & 0.0341 & 0.0282 & 0.0287 & 0.0056 & 0.0456 \\
\hline
\end{tabular}

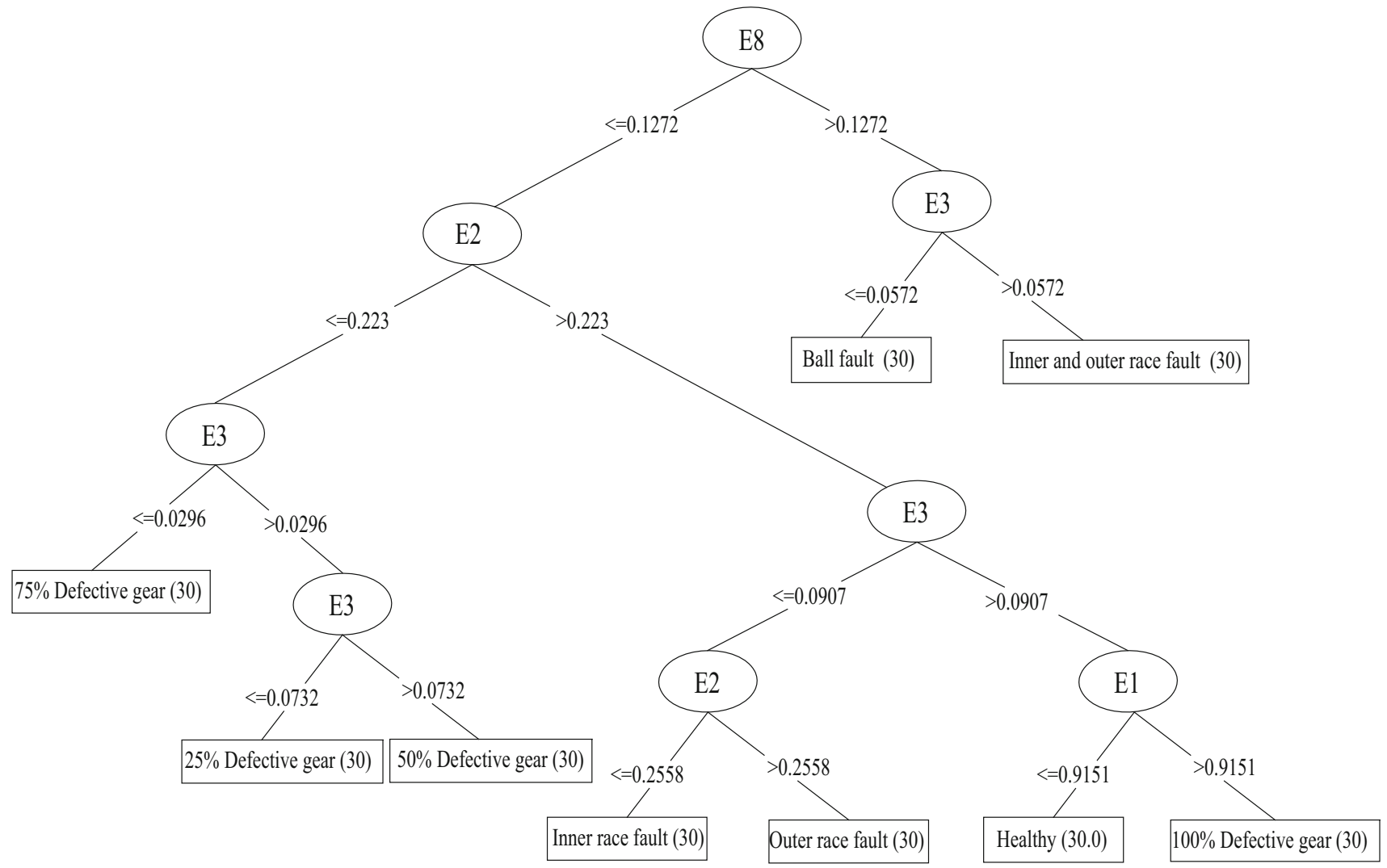

Figure 9. Decision tree.

shows that four best features for classification features presented visually are $E 1, E 2, E 3$ and $E 8$ in the order of importance. The important features were selected based on the information gain and entropy available in each EMD feature. The details about information gain and entropy reduction was explained in section 5.1. The features that provided more 
Table 3. Confusion matrix.

\begin{tabular}{lrrrrrrrrc}
\hline $\mathrm{a}$ & $\mathrm{b}$ & $\mathrm{c}$ & $\mathrm{d}$ & $\mathrm{e}$ & $\mathrm{f}$ & $\mathrm{g}$ & $\mathrm{h}$ & $\mathrm{i}$ & $\begin{array}{c}\text { Engine gearbox } \\
\text { classes }\end{array}$ \\
\hline 30 & 0 & 0 & 0 & 0 & 0 & 0 & 0 & 0 & $\mathrm{a}-$ Healthy \\
0 & 30 & 0 & 0 & 0 & 0 & 0 & 0 & 0 & $\mathrm{~b}-25 \%$ faulty gear \\
0 & 0 & 30 & 0 & 0 & 0 & 0 & 0 & 0 & $\mathrm{c}-50 \%$ faulty gear \\
0 & 0 & 0 & 30 & 0 & 0 & 0 & 0 & 0 & $\mathrm{~d}-75 \%$ faulty gear \\
1 & 0 & 0 & 0 & 29 & 0 & 0 & 0 & 0 & $\mathrm{e}-100 \%$ faulty gear \\
0 & 0 & 0 & 0 & 0 & 30 & 0 & 0 & 0 & $\mathrm{f}-$ Outer race fault \\
0 & 0 & 0 & 1 & 0 & 0 & 29 & 0 & 0 & $\begin{array}{c}\text { g- ORF) } \\
\text { (IRF) race fault }\end{array}$ \\
1 & 0 & 0 & 0 & 0 & 0 & 0 & 29 & 0 & $\mathrm{~h}-$ Inner outer race \\
fault
\end{tabular}

information about the engine gearbox condition were selected by the decision tree. From figure $9, E 8$ is the root node. Based on the feature $E 8$, the decision tree is formed in such a way that when the $E 8$ feature value is greater than 0.1272 and $E 3$ is less than or equal to 0.0572 , then it is classified as 'ball fault' condition. When the $E 8$ feature value is greater than 0.1272 and $E 3$ is greater than 0.0572 , then it is classified as 'inner and outer race fault' condition and so on. The selected features were used for classification of engine gearbox using Naïve Bayes algorithm.

\subsection{Classification using Nä̈ve Bayes algorithm}

Table 3 shows the confusion matrix produced by Naïve Bayes classifier. Diagonal elements in this matrix indicate correctly classified samples.

- Consider the first row: the first element represents the samples that belong to the 'healthy' class. The second element in first row represents the samples that belong to the 'healthy' class but misclassified as '25\% faulty' gear category. The third element in first row indicates the 'healthy' samples misclassified as '50\% faulty' gear condition and so on.

- From this table it can be noticed that, out of 270 samples pertaining to each class of gear and bearings, three samples have been misclassified. Therefore, the classification accuracy of about $98.88 \%$ was provided by the Naïve Bayes classifier with EMD features in fault diagnosis of engine gearbox.

\section{Conclusion}

This investigation presents fault diagnosis of engine gearbox using machine learning approach based on vibration signals. This methodology involved collecting 30 acceleration vibration signal samples for nine different classes of mechanical machine elements, specifically gears and bearings. The acquired signals were decomposed into first eight IMFs using the EMD method. The energy feature vector was evaluated and treated as an input vector to $\mathrm{J} 48$ algorithm (decision tree) for the most important feature selection. Naïve Bayes classifier was used for fault classification. Classification accuracy was found to be $98.88 \%$, which is reasonably good. Based on the results obtained, the proposed methodology with machine learning techniques can be recommended for practical applications and development of on-line fault diagnosis systems for machine condition monitoring.

\section{Acknowledgements}

The authors acknowledge the funding support from SOLVE: The Virtual Lab @ NITK (www.solve.nitk.ac.in) and experimental facility provided by the Centre for System Design (CSD): A Centre of Excellence at NITK-Surathkal. The authors also acknowledge the help rendered by Dr. V. Sugumaran, Associate Professor, VIT University, Chennai.

\section{References}

[1] Vernekar K, Kumar H and Gangadharan K V 2014 Gear fault detection using vibration analysis and continuous wavelet transform. Procedia Mater. Sci. 5: 1846-1852

[2] Chen F, Tang B and Chen R 2013 A novel fault diagnosis model for gearbox based on wavelet support vector machine with immune genetic algorithm. Measurement 46: 220-232

[3] Yu Y and Junsheng C 2006 A roller bearing fault diagnosis method based on EMD energy entropy and ANN. J. Sound. Vib. 294: 269-277

[4] Kumar H, Ranjit Kumar T A, Amarnath M and Sugumaran V 2014 Fault diagnosis of bearings through vibration signal using Bayes classifiers. Int. J. Comput. Aid. Eng. Technol. 6: $14-28$

[5] Addin O, Sapuan S M, Mahdi E and Othman M 2007 A Naïve-Bayes classifier for damage detection in engineering materials. Mater. Des. 28: 2379-2386

[6] Muralidharan V and Sugumaran V 2012 A comparative study of Naïve Bayes classifier and Bayes net classifier for fault diagnosis of monoblock centrifugal pump using wavelet analysis. Appl. Soft. Comput. 12: 2023-2029

[7] Vedant, Sugumaran V, Amarnath M and Kumar H 2013 Fault diagnosis of helical gear box using sound signal using Naïve Bayes and Bayes net. Int. J. Eng. Technol. Res. 1: 98-105

[8] Addin O, Sapuan S M, Othman M and Ali B A 2011 Comparison of Naïve Bayes classifier with back propagation neural network classifier based on f-folds feature extraction algorithm for ball bearing fault diagnostic system. Int. J. Phys. Sci. 6: 3181-3188 
[9] Sharma A, Sugumaran V and Babu Devasenapati S 2014 Misfire detection in an IC engine using vibration signal and decision tree algorithms. Measurement 50: 370-380

[10] Hemantha Kumar T A, Ranjit Kumar M, Amarnath M and Sugumaran V 2012 Fault diagnosis of antifriction bearings through sound signals using support vector machine. $J$. Vibroeng. 14: 1601-1606

[11] Saimurugan M, Ramachandran K I, Sugumaran V and Sakthivel N R 2011 Multi-component fault diagnosis of rotational mechanical system based on decision tree and support vector machine. Expert. Syst. Appl. 38: 3819-3826

[12] Sugumaran V and Ramachandran K I 2007 Automatic rule learning using decision tree for fuzzy classifier in fault diagnosis of roller bearing. Mech. Syst. Signal Process. 21: 2237-2247

[13] Sun W, Chen J and Li J 2007 Decision tree and PCA-based fault diagnosis of rotating machinery. Mech. Syst. Signal Process. 21: 1300-1317
[14] Wang Y S, Ma Q H, Zhu Q, Liu X T and Zhao L H 2014 An intelligent approach for engine fault diagnosis based on Hilbert-Huang transform and support vector machine. Appl. Acoust. 75: 1-9

[15] Lin J and Qu L 2000 Feature extraction based on Morlet wavelet and its application for mechanical fault diagnosis. J. Sound. Vib. 234: 135-148

[16] Peng Y H, Flach P A, Brazdil P and Soares C 2012 Decision tree-based data characterization for meta-learning. In: Proceedings of the IDDM Workshop, Finland

[17] Sugumaran V, Muralidharan V and Ramachandran K I 2007 Feature selection using decision tree and classification through proximal support vector machine for fault diagnostics of roller bearing. Mech. Syst. Signal Process. 21: 930-942

[18] Girish Kumar M, Hemantha K, Gangadhar N, Kumar H and Krishna P 2014 Fault diagnosis of welded joints through vibration signals using Naïve Bayes algorithm. Procedia Mater. Sci. 5: 1922-1928 